\title{
Perceptions du personnel des centres de réadaptation en déficience intellectuelle et troubles envahissants du développement quant aux troubles du comportement chez les jeunes enfants \\ Perceptions of workers of rehabilitation centers on problem behavior in young children
}

\author{
Mélina Rivard, Carmen Dionne, Diane Morin et Marc-André Gagnon
}

Volume 42, numéro 1, 2013

URI : https://id.erudit.org/iderudit/1061726ar

DOI : https://doi.org/10.7202/1061726ar

Aller au sommaire du numéro

Éditeur(s)

Revue de Psychoéducation

ISSN

1713-1782 (imprimé)

2371-6053 (numérique)

Découvrir la revue

Citer cet article

Rivard, M., Dionne, C., Morin, D. \& Gagnon, M.-A. (2013). Perceptions du personnel des centres de réadaptation en déficience intellectuelle et troubles envahissants du développement quant aux troubles du comportement chez les jeunes enfants. Revue de psychoéducation, 42(1), 115-133.

https://doi.org/10.7202/1061726ar

\section{Résumé de l'article}

La présence de troubles du comportement (TC) chez les enfants ayant un trouble du spectre de l'autisme (TSA) comporte de nombreux défis pour les différentes personnes appelées à interagir avec eux, telles que leurs familles, leurs pairs et leurs intervenants. Cependant, dans le domaine des TSA, ce phénomène demeure peu étudié pendant la période de développement couvrant la petite enfance et peu d'outils adaptés sont disponibles pour les milieux de pratique. Cette étude est une enquête réalisée à l'aide d'un questionnaire qui vise à recenser les besoins perçus par les intervenants, professionnels et gestionnaires travaillant dans les programmes d'intervention comportementale intensive (ICI) en centre de réadaptation en déficience intellectuelle et troubles envahissants du développement (CRDITED) quant aux TC chez les enfants recevant leurs services. La majorité des répondants (95,5\%) indiquent avoir travaillé avec au moins un enfant présentant des TC au cours de la dernière année. Ceux-ci font ressortir des besoins sur le plan de la formation, de l'évaluation, de l'intervention et du soutien à l'intégration de ces enfants dans les milieux de garde et préscolaires.
Tous droits réservés ( $\odot$ La Corporation de la Revue Canadienne de Psycho-Éducation, 2013
Ce document est protégé par la loi sur le droit d'auteur. L'utilisation des services d'Érudit (y compris la reproduction) est assujettie à sa politique d'utilisation que vous pouvez consulter en ligne. 


\section{Perceptions du personnel des centres de réadaptation en déficience intellectuelle et troubles envahissants du développement quant aux troubles du comportement chez les jeunes enfants}

\section{Perceptions of workers of rehabilitation centers on problem behavior in young children}

M. Rivard ${ }^{1}$
C. Dionne
D. Morin ${ }^{1}$
M.-A. Gagnon ${ }^{1}$

1 Université du Québec à Montréal

2 Université du Québec à Trois-Rivières

\section{Correspondance :}

Mélina Rivard

Université du Québec à Montréal C.P. 8888 , Succ. Centre-Ville Montréal (Québec) H3C 3P8 rivard.melina@uqam.ca

\section{Résumé}

La présence de troubles du comportement (TC) chez les enfants ayant un trouble du spectre de l'autisme (TSA) comporte de nombreux défis pour les différentes personnes appelées à interagir avec eux, telles que leurs familles, leurs pairs et leurs intervenants. Cependant, dans le domaine des TSA, ce phénomène demeure peu étudié pendant la période de développement couvrant la petite enfance et peu d'outils adaptés sont disponibles pour les milieux de pratique. Cette étude est une enquête réalisée à l'aide d'un questionnaire qui vise à recenser les besoins perçus par les intervenants, professionnels et gestionnaires travaillant dans les programmes d'intervention comportementale intensive $(\mathrm{ICl})$ en centre de réadaptation en déficience intellectuelle et troubles envahissants du développement (CRDITED) quant aux TC chez les enfants recevant leurs services. La majorité des répondants $(95,5 \%)$ indiquent avoir travaillé avec au moins un enfant présentant des TC au cours de la dernière année. Ceux-ci font ressortir des besoins sur le plan de la formation, de l'évaluation, de l'intervention et du soutien à l'intégration de ces enfants dans les milieux de garde et préscolaires.

Mots-clés: troubles du comportement, petite enfance, trouble du spectre de l'autisme, perception des besoins

Note de l'auteur: Nous tenons à remercier le Fonds de recherche du Québec - Société et culture pour son appui financier (bourse postdoctorale) à la réalisation de ce projet. Nous exprimons également notre gratitude aux CRDITED et aux personnes ayant participé à notre projet. Leur enthousiasme et leur collaboration nous ont démontré qu'ils percevaient réellement la pertinence de cette recherche. Nous voulons également remercier $\mathrm{M}^{\mathrm{me}}$ Claire Robitaille pour son aide lors de la révision linguistique de cet article. Le devis de recherche et un court résumé des résultats préliminaires ont fait l'objet de différentes présentations orales lors du congrès et d'un acte de colloque pour la Revue francophone en déficience intellectuelle. Un deuxième article sur cette enquête sera également publié. Cet article comprend les résultats concernant les pratiques d'évaluation et d'intervention des intervenants concernant les TC. 


\begin{abstract}
The presence of behavioral problems $(B P)$ in young children with Autism Spectrum Disorder (ASD) presents many challenges for the persons in their entourage, as their parents, peers and their therapists. However, it remains that there is few studies on this phenomenon in the field of early childhood and ASD and practice settings have few tools of evaluation and intervention adapted to this population. This study is a survey conducted by the bias of a questionnaire designed to identify the needs perceived by therapists, professionals and managers working intensive behavioral intervention (IBI) programs in a rehabilitation center for ID and ASD according the presence of BP in children receiving their services. The vast majority of respondents (95.5\%) reported having worked with at least one child with BP in the past year. They highlight the needs in terms of training, assessment, intervention and the integration of these children.
\end{abstract}

\title{
Key words: Behavior Problems, Early Childhood, Autism Spectrum Disorders, Perceptions of Needs
}

\section{Introduction}

La présence de troubles du comportement (TC) chez les jeunes enfants ayant un trouble du spectre de l'autisme (TSA) comporte de nombreux défis pour les familles ainsi que pour les milieux d'intégration et d'intervention. Pour ces derniers, les intervenants œuvrant auprès de personnes ayant des TC vivent davantage de stress, d'épuisement professionnel et d'accidents de travail (Hastings \& Brown, 2002; Mitchell \& Hastings, 2001; Tassé, Sabourin, Garcin, \& Lecavalier, 2010). En ce qui a trait plus spécifiquement à la petite enfance dans le domaine des TSA, les intervenants mentionnent que les TC constituent un obstacle à l'efficacité des programmes d'intervention comportementale intensive (ICI) (Symes, Remington, Brown, \& Hasting, 2006). Notons que ces programmes sont ceux privilégiés dans les centres de réadaptation en déficience intellectuelle et troubles envahissants du développement (CRDITED) pour la population d'enfants âgés de 0 à 6 ans ayant un TSA. II n'y a cependant pas de données disponibles sur le nombre d'enfants qui présentent à la fois un TSA et un TC et à qui on offre des services d'ICl en CRDITED, pas plus qu'on ne dispose d'informations sur les façons dont les services leur sont individualisés. L'importance de cibler les TC dès le plus jeune âge est de plus en plus mise en évidence dans le domaine des TSA, mais ce phénomène demeure peu étudié directement pendant la période de développement couvrant la petite enfance (Fox, Dunlap, \& Cushing, 2002). II ressort de la littérature scientifique que peu d'outils d'identification et d'intervention adaptés sont disponibles pour cette population (Farmer \& Aman, 2011; Mahan \& Matson, 2011). Compte tenu de la couverture universelle des programmes ICI dans les CRDITED du Québec pour les enfants ayant un TSA, des possibles effets des TC sur la réponse aux services et du besoin de disposer d'outils adaptés pour cette clientèle, il devient important d'étudier ce phénomène dans les milieux qui reçoivent ces enfants et de documenter les perceptions des personnes intervenant directement auprès d'eux quant aux besoins entourant les TC. 


\section{Prévalence des TC chez les enfants ayant un TSA}

Quoiqu'il y ait peu d'études systématiques portant sur la prévalence des TC chez les enfants ayant un retard global de développement d'âge préscolaire (Emerson \& Einfeld, 2010), on reconnaît que ces troubles sont plus fréquents chez les enfants ayant une déficience intellectuelle (DI) ou un TSA (Baker, Blacher, Crnic, \& Edelbrock, 2002; Matson, Gonzalez, Wilkins, \& Rivet, 2008). Pour les enfants ayant un TSA, sans être un indicateur précis de la prévalence des TC à l'âge préscolaire, une étude rapporte que parmi 182 enfants âgés de 2 à 17 ans, $93,4 \%$ participants présentent au moins une forme de TC (Matson, Wilkins, \& Macken, 2009). Ce pourcentage élevé a été confirmé dans une étude récente de Jang, Dixon, Tarbox et Granpeesheh (2011). D'autres travaux soulèvent que plus de $60 \%$ des enfants ayant un diagnostic de TSA présenteraient des TC (Gurny, McPheeters, \& Davis, 2006; Icasiano, Hewson, Machet, Cooper, \& Marshall, 2004). II n'y a toutefois pas d'étude qui établit clairement, durant la période préscolaire, le pourcentage d'enfants ayant un TSA qui manifestent aussi des comportements problématiques dont la fréquence et l'intensité sont telles qu'ils se distinguent des symptômes définis dans les TSA.

Les études empiriques montrent que les comportements problématiques les plus communs chez les enfants ayant une DI ou un TSA sont : les comportements d'agressivité physique envers autrui, les comportements destructeurs, les crises, l'agressivité verbale, les comportements stéréotypés et les comportements d'automutilation (Horner, Carr, Stram, Todd, \& Reed, 2002; Machalicek, O'Reilly, Beretvas, Sigafoos, \& Luancioni, 2007).

La période préscolaire serait celle la plus à risque pour le développement de comportements problématiques pour l'ensemble de la population et leur présence en bas âge serait un prédicteur de comportements agressifs et de différentes conséquences négatives durant la vie de la personne (voir Emerson \& Einfeld, 2010). Aussi, en l'absence d'intervention appropriée, les TC chez les enfants ayant une DI ou un TSA perdureraient et pourraient s'exacerber (Emerson \& Einfeld, 2010). II devient ainsi important d'en tenir compte de façon spécifique lors d'interventions en petite enfance dans les CRDITED afin de prévenir la cristallisation de ces troubles à l'âge adulte.

\section{La formation et les perceptions face aux troubles du comportement}

Les perceptions du personnel œuvrant auprès de la population ayant une DI ou un TSA face aux TC auraient une incidence importante sur le développement et le maintien des TC (Hastings, 2005). Des études rapportent que les perceptions et les attributions causales des parents et des intervenants par rapport aux TC influencent les stratégies et les pratiques d'intervention utilisées auprès des enfants (Jacobs \& Woolfson, 2012). L'expérience et le niveau de connaissances seraient deux facteurs qui ont des effets sur l'évaluation des causes des TC et l'utilisation des stratégies d'intervention efficaces chez le personnel (Hastings, 1997). Après avoir reçu la formation appropriée, le personnel utiliserait davantage certaines stratégies d'intervention appuyées par les données probantes dont celles basées sur l'analyse fonctionnelle (Berryman, Evans, \& Kalbag, 1994). Les travaux dans 


\section{8}

le domaine (voir notamment Hasting, 1997) font ainsi ressortir l'importance de la formation dans la compréhension des fonctions et des causes des TC chez le personnel. La formation permet dès lors l'adoption de meilleures stratégies d'intervention en regard des TC, stratégies d'intervention qui sont préventives et orientées vers les causes. La formation serait une bonne façon d'intervenir sur les croyances du personnel, permettant ainsi d'influencer l'utilisation qu'on fait des stratégies d'intervention appropriées (leurs comportements) (Allen \& Tynan, 2000; Hastings, 1997; Morgan \& Hastings, 1998). L'ensemble des travaux sur les effets de la formation et la compréhension du personnel quant aux causes des TC fait ressortir l'importance de documenter deux aspects de la question: les types de formation reçue en regard des $\mathrm{TC}$ dans les équipes $\mathrm{ICl}$ intervenant auprès des enfants et leurs perceptions par rapport aux défis associés à l'intervention auprès de ces enfants

\section{Les troubles du comportement et l'intervention comportementale intensive}

Des études réalisées au Royaume-Uni auprès des familles révèlent que les enfants ayant des TC sont souvent exclus des différents services qui sont offerts aux enfants ayant une DI ou un TSA mais qui n'ont pas de TC (McGill, Papachristoforou, \& Cooper, 2006; Wodehouse \& McGill, 2009). Ces familles rapportent des insatisfactions quant aux services qu'ils reçoivent, notamment au regard de l'inefficacité des stratégies d'intervention utilisées et du manque d'expertise des intervenants concernant les TC (voir Wodehouse \& McGill, 2009). En outre, selon une étude réalisée aux États-Unis par Symes, Remington, Brown et Hasting (2006), les éducateurs offrant des services d'ICl à des enfants ayant un TSA rapportent que la présence de TC est un facteur qui nuit à l'efficacité de cette intervention. Une autre étude de Rivard, Forget, Giroux, Regli, \& Kerr, soumis (voir Rivard, 2011) réalisée au Québec en CRDITED montre que la présence de comportements problématiques chez les enfants ayant un TSA peut nuire à la progression du langage et des habiletés sociales en cours d'ICl.

II faut savoir que les programmes d'ICI faisant l'objet de manuels dont ceux de Leaf et McEachin (1999) et de Maurice (1996), incluent de façon systématique des éléments d'intervention qui ciblent les comportements problématiques à même les composantes essentielles de l'intervention. Néanmoins, compte tenu de l'implantation relativement récente des services d'ICI dans les CRDITED du Québec, de l'hétérogénéité des modèles utilisés dans ces différents centres et de la variation quant à leur niveau d'implantation (voir Gamache, Joly, \& Dionne, 2010), nous avons peu d'information sur les éléments d'intervention reliés aux comportements problématiques qui sont utilisés de façon spécifique auprès des enfants. En effet, il semble que certaines cibles d'intervention (p. ex., travailler sur les apprentissages de type préscolaire plutôt que de travailler à l'acquisition des demandes spontanées chez l'enfant et qui sont en lien avec ses besoins) ou certaines stratégies (p. ex., l'enseignement par essais distincts par opposition à l'enseignement fortuit ou incident) sont privilégiées lors des récents programmes d'ICI en CRDITED (voir Rivard \& Forget, 2012). Cela peut s'expliquer par plusieurs facteurs, notamment par le fait que les enfants reçoivent un nombre d'heures relativement restreint (moins de 20 heures par semaine), pour une durée d'intervention relativement courte compte tenu de l'âge tardif où les enfants reçoivent le diagnostic et enfin, en raison des listes 
d'attente pour l'obtention de services. Compte tenu de l'entrée imminente de l'enfant à l'école, la priorité d'intervention étant alors accordée aux apprentissages de type préscolaire. Cela peut aussi s'expliquer par le type de formation que les intervenants des programmes d'ICI reçoivent. Nous avons actuellement peu d'information sur le contenu des formations offertes au regard des différentes stratégies d'intervention incluses dans les programmes d'ICI telles que l'enseignement par essais distincts, l'enseignement fortuit ou incident et les stratégies d'évaluation qui devraient être utilisées pour l'analyse des comportements problématiques telle que l'évaluation fonctionnelle. Des constats similaires ont aussi été faits lors du congrès annuel 2012 de l'Association internationale pour l'analyse du comportement en autisme (Association for Behavior Analysis International (ABAI) AUTISM; voir présentation de Horner, 2012). Par ailleurs, l'enseignement par essais distincts a été privilégié dans les programmes d'ICI depuis leur implantation ou mise en oeuvre dans les milieux de pratique et ce, d'autant plus que ce type d'enseignement est efficace et que la formation aux intervenants pour son utilisation auprès des enfants est relativement simple et efficiente. On reconnaît néanmoins l'importance d'utiliser d'autres stratégies découlant de l'analyse appliquée du comportement (AAC) dans le cadre des programmes d'ICl, afin de maximiser les gains réalisés par les enfants (voir Rivard \& Forget, 2012; Fava \& Strauss, 2011).

Les questions de TC et d'intervention précoce sont ainsi deux priorités établies par les CRDITED du Québec et sont dans deux guides de pratique distincts proposés par la Fédération québécoise des CRDITED. Le premier guide porte sur les troubles graves du comportement (TGC) et le second, sur l'intervention auprès des enfants ayant un TSA âgés de 0 à 5 ans (FQCRDITED, rapport annuel 2009-2010). L'implantation de ces deux guides a été mise de l'avant au sein des établissements de services. Nous avons cependant peu d'informations sur l'intervention relative aux TC auprès des jeunes enfants ayant des TSA. II n'y a pas actuellement d'information disponible au Québec sur le nombre d'enfants d'âge préscolaire présentant des TC qui sont desservis dans les CRDITED, ou encore sur les services qu'ils reçoivent. La présente étude est une démarche exploratoire pour documenter la situation des enfants présentant un TSA et qui présentent des TC selon la perception des personnes travaillant dans les programmes d'ICl en CRDITED. L'étude cherche d'abord à savoir si les éducateurs, les professionnels et les gestionnaires des services d'ICI reconnaissent travailler avec des enfants ayant des TC. Des informations sont colligées sur les types de TC qu'ils perçoivent comme étant les plus problématiques pour l'ICl. Toujours selon leur point de vue, les rôles qu'ils assument auprès de ces enfants sont également documentés. Finalement, des données sont recueillies sur leurs perceptions de l'intégration de ces enfants dans les milieux d'accueil, tels que les milieux de garde et scolaire.

\section{Objectifs}

L'objectif général de cette étude est de dresser un portrait des perceptions des éducateurs, des professionnels et des gestionnaires travaillant dans les programmes d'ICI en CRDITED par rapport aux TC chez les jeunes enfants. Quatre questions de recherche précisent cet objectif : 1) les équipes spécialisées en ICI dans les CRDITED perçoivent-elles recevoir dans leurs services des enfants présentant des TC? 2) quels comportements problématiques sont rapportés par les 
répondants chez ces enfants? 3) les répondants observent-ils l'intégration de ces enfants en milieux de garde et scolaire ordinaires et quelles sont leur perception des besoins par rapport à une intégration réussie de ces enfants? 4) quelle est la perception des répondants par rapport à leur rôle auprès de ces enfants?

Cette étude est réalisée sous forme d'enquête dont les données sont recueillies à l'aide d'un questionnaire s'adressant aux intervenants (éducateurs, professionnels et gestionnaires) des CRDITED du Québec.

\section{Méthode}

Cette étude a été approuvée par le Comité éthique de la recherche conjoint (CÉRC) des CRDITED du Québec.

\section{Participants}

L'échantillon est constitué de 44 éducateurs, professionnels ou gestionnaires travaillant dans sept CRDITED. Les CRDITED offrent des services spécialisés de deuxième ligne aux personnes ayant une DI ou un TSA. Selon l'article 84 de la Loi sur les services de santé et les services sociaux:

« La mission d'un CRDI est d'offrir des services d'adaptation ou de réadaptation et d'intégration sociale à des personnes qui, en raison de leur déficience intellectuelle (...) requièrent de tels services, de même que des services d'accompagnement et de support à l'entourage de ces personnes. À cette fin, l'établissement qui exploite un tel centre reçoit, sur référence, les personnes présentant une déficience intellectuelle; il s'assure que leurs besoins soient évalués et que les services requis leur soient offerts à l'intérieur de ses installations ou dans leur milieu de vie, à l'école, au travail ou à domicile ou, si nécessaire, il s'assure qu'elles soient dirigées le plus tôt possible vers les centres, les organismes ou les personnes les plus aptes à leur venir en aide $"$.

Ces services sont dispensés selon divers programmes qui diffèrent en fonction de l'âge et des besoins cliniques de la personne et aussi selon l'organisation des services de chacun des CRDITED. Parmi ces programmes, les programmes d'ICl offerts à la petite enfance pour les enfants ayant un TSA sont à l'étude. Ces programmes d'intervention, découlant de l'analyse appliquée du comportement, visent à susciter chez l'enfant l'apprentissage de comportements instrumentaux, sociaux et cognitifs permettant d'améliorer son intégration scolaire et sociale. Comme le propose le National Research Council (2001), les programmes d'ICl sous-tendent également divers paramètres d'intervention, soit : un début précoce; un engagement actif (un minimum de 20 heures, 5 jours par semaine, durant toute l'année); l'utilisation d'un curriculum structuré et planifié qui inclut des objectifs spécifiques; dans les interventions, une priorité accordée à l'enseignement direct des habiletés de base (telles que la communication, les compétences sociales, le développement cognitif, les habiletés de jeu et la gestion des comportements inadéquats); des procédures de généralisation des acquis et de maintien des habiletés; des interventions individualisées; un faible ratio 
intervenant-enfant (idéalement un ratio intervenant-enfant un pour un); l'implication des parents; la formation spécialisée des intervenants et l'évaluation continue des progrès. Néanmoins, il faut savoir qu'actuellement, il n'y a pas de directive claire ou d'implantation uniforme de ces programmes dans les différents CRDITED. Nous avons peu d'information sur les composantes d'intervention implantées auprès des enfants dans le réseau public des CRDITED.

Les CRDITED ont été invités à participer au projet lors d'une rencontre de la Table des affaires professionnelles qui regroupe les directeurs des services professionnels des CRDITED. Une description du projet ainsi que le questionnaire ont été déposés. Huit CRDITED ont accepté de participer (taux d'acceptation de $44,4 \%$ ). Un des CRIDTED ayant accepté de participer à l'étude ne fait pas partie de la présente analyse, ne desservant pas la population ayant un TSA. Les participants forment un échantillon de convenance, déterminé par le type d'organisation de services en CRDITED et par le consentement des participants. Le recrutement des participants a été initié par les coordonnateurs de la recherche ou les personnes ayant ce rôle au sein des CRDITED. Parmi chacune des équipes spécialisées en ICI des CRDITED participants, un coordonnateur ou chef de services, un professionnel et un éducateur ont été sollicités. Le nombre de participants par CRDITED diffère selon la configuration des services de chacun des établissements participants. Parmi les participants, $41 \%$ travaillent en Montérégie, $14 \%$ dans la région de Montréal, $14 \%$ à Trois-Rivières, $11 \%$ en Estrie, $11 \%$ à Québec, $7 \%$ dans la région de Chaudières-Appalaches. L'échantillon couvre ainsi $41 \%$ des régions administratives du Québec.

Parmi les personnes ayant accepté de participer à l'étude, on retrouve 26 éducateurs, 13 professionnels et 5 gestionnaires. Parmi les répondants, 24 personnes ont jusqu'à cinq ans d'expérience en CRDITED, 15 ont entre six et dix ans d'expérience et 5 ont au moins 11 ans d'expérience en CRDITED.

\section{Outil de mesure}

Un questionnaire autoadministré et semi-structuré a été construit pour les fins de l'enquête afin de permettre de documenter les questions de recherche énoncées précédemment. Les questions incluses dans ce questionnaire proviennent d'une analyse des écrits scientifiques portant sur les TC et sur les difficultés observées quant à l'évaluation, l'intervention et l'intégration des enfants ayant une DI ou un TSA et des TC. Les deux cochercheurs du projet ont réalisé une validation indépendante du questionnaire. Ce dernier a alors été mis à l'essai lors d'un prétest auprès d'une éducatrice et d'une psychologue travaillant en CRDITED. Au total, le questionnaire comprend 24 questions ouvertes. Comme l'objectif du questionnaire est de faire ressortir les perceptions des intervenants et leurs besoins tels qu'ils les vivent au quotidien dans leur travail avec les enfants ayant un TC, des questions ouvertes devaient être privilégiées pour faire ressortir leurs préoccupations principales. Certaines questions, concernant davantage les thèmes de la formation, de l'évaluation et de l'intervention, sont couvertes dans un autre article. En plus de données sociodémographiques, les questions retenues dans le cadre de cet article permettent de recueillir des informations sur : les observations des répondants par rapport au fait qu'ils reçoivent ou non des enfants dans leurs 
services qui correspondent à la définition des TC par Tassé et ses collègues (2010), les types de comportements problématiques présentés et perçus comme les plus perturbateurs par ces enfants et le rôle joué par les répondants auprès de ces enfants. La terminologie utilisée dans cette étude pour définir les TC (Tassé et al., 2010) est celle qui est actuellement privilégiée par la FQCRDITED et le Service québécois d'expertise en TGC (SQETGC). Selon cette définition :

Action ou ensemble d'actions qui est jugé problématique parce qu'il s'écarte des normes sociales, culturelles ou développementales et qui est préjudiciable à la personne ou à son environnement social ou physique. Ce trouble est jugé grave s'il met en danger, réellement ou potentiellement, l'intégrité physique ou psychologique de la personne, d'autrui ou de l'environnement, ou qu'il compromet sa liberté, son intégration ou ses liens sociaux (p.68).

Des questions relatives à l'intégration de ces enfants et aux défis associés à leur intégration sont aussi formulées. Une dernière question permet aux participants d'émettre des commentaires généraux concernant les besoins en TC dans le domaine des TSA et de la petite enfance.

\section{Procédure}

Le recrutement a été initié, dans un premier temps, par le responsable de la recherche de chacun des CRDITED. Ce dernier remettait le formulaire de consentement et le questionnaire aux participants potentiels de chacune des équipes (petite enfance et TGC). Les personnes intéressées à participer transmettaient par la suite les documents complétés (formulaire de consentement et questionnaire) directement aux chercheurs ou par le biais du responsable de la recherche de leur établissement. Les participants pouvaient communiquer avec les chercheurs s'ils avaient besoin de soutien pour compléter le questionnaire autoadministré, ce qu'ont fait trois répondants. Les questionnaires ont été dénominalisés pour assurer la confidentialité et ensuite identifiés par un code alphanumérique.

\section{Analyses}

Les données des questionnaires ont été analysées à partir d'une grille de codification qui permet de traiter l'information selon les questions de recherche. Pour chacune des questions, des catégories de réponses ont été préétablies pour regrouper l'information et comptabiliser le nombre de personnes ayant répondu dans le sens de chacune des catégories identifiées. À l'aide d'une première version de la grille, dix questionnaires complétés par les répondants ont été codifiés par deux personnes différentes pour tester l'outil. À la suite de cette mise à l'essai, de nouvelles catégories ont été identifiées pour permettre une meilleure représentativité des réponses des participants. Les résultats sont ainsi présentés selon le nombre de personnes par catégorie de réponse. Afin d'appuyer les résultats, les propos de certains participants sont aussi inclus. Des données quantitatives et qualitatives ont donc été utilisées pour présenter les résultats. Un doctorant en psychologie a réalisé la codification et la synthèse des données. L'accord interjuge visant à assurer la fiabilité des données a été mesuré par la concordance observée lors de 
la codification de dix questionnaires. L'accord interjuge par rapport à la codification des données du questionnaire est de $100 \%$ pour ces dix questionnaires.

\section{Résultats}

\section{Enfants âgés de 6 ans et moins ayant des TC desservis en CRDITED}

Pour répondre à la première question de recherche, les participants ont été invités à estimer le nombre d'enfants qui correspondent à la définition des TC selon Tassé et ses collaborateurs (2010) et avec lesquels ils ont travaillé lors de la dernière année. Pour ce faire, on demandait aux participants de consulter le dossier clinique de chacun de ces enfants et de répondre conformément aux informations contenues au dossier. Cette démarche ne visait pas à identifier le nombre d'enfants ayant des TC dans les services par rapport au nombre d'enfants total, mais plutôt d'évaluer si les répondants perçoivent recevoir des enfants ayant de tels comportements dans leur service. Au total, $95,5 \%$ des 44 répondants rapportent avoir travaillé avec au moins un enfant ayant des TC au cours de la dernière année. De manière plus spécifique, plus du quart des répondants $(27,3 \%)$ rapportent avoir travaillé avec un enfant ayant ces troubles au cours de la dernière année, $38,6 \%$, avec deux à quatre enfants tandis que $29,5 \%$ ont travaillé avec cinq enfants ou plus.

Un répondant mentionne qu'il est : « difficile de dire le nombre d'enfants [ayant des TC] parce qu'ils n'ont pas de méthode de recensement. II n'y a pas non plus de façon de compiler les différents comportements ».

Un répondant indique également : « Pendant longtemps, les TGC n'étaient pas reconnus chez les enfants. Les enfants ne sont jamais identifiés TGC avant l'âge de 6 ans, ils ne le sont que lorsqu'ils passent dans le service destiné aux 6 ans et plus. En bas de 6 ans, les TGC sont considérés comme des caprices, ce n'est que lorsque l'enfant vieillit (plus fort, plus lourd) que le TGC est identifié ». Un participant souligne d'ailleurs qu'en CRDITED, dans les services d'intervention précoce : « Les intervenants n'ont pas le réflexe de demander des services [de l'équipe spécialisée en TC] ». Pourtant, tel que rapporté par un autre participant : " Il est important de comprendre que les TC rajoutent des problématiques aux enfants ayant un TSA et qu'il faut travailler pour prévenir ». Dans le même sens, un participant signale : " II arrive régulièrement qu'on doive travailler sur les TC de l'enfant pour être en mesure d'intervenir efficacement en ICl ». Un autre participant fait ressortir aussi : « II faut intervenir sur les TC avant de pouvoir faire de I'ICl et les milieux ont beaucoup d'attentes envers les CRDITED parce qu'ils sont en mode survie. La coordination des services et l'application du PI [plan d'intervention] deviennent lourdes pour l'intervenant responsable du dossier ». D'ailleurs, cinq répondants font remarquer qu'ils doivent travailler sur les TC avant de pouvoir amorcer l'enseignement et les programmes d'intervention, dont l'ICl. 


\section{Types de comportements problématiques}

La deuxième question de recherche concerne les types de comportements présentés par les enfants ayant des TC/TGC lors de la dernière année et qui semblent être les plus problématiques pour l'intervention. Les comportements suivants ont été mentionnés : agression physique envers autrui (43 mentions); comportements perturbateurs (41); comportements d'automutilation (29); non collaboration (29); agitation (29); troubles de l'alimentation (24); stéréotypies (20); provocation (19); difficultés dans la gestion des émotions (18); troubles du sommeil (17); problématique en lien avec la propreté (17); comportements destructeurs (16); autres (p. ex., pica, comportements à caractère sexuel) (15); troubles anxieux (12); retrait social - isolement (8); fugue (6) et tics (5). Quatre répondants soulignent aussi qu'il serait difficile de déterminer ce qui appartient au TSA aux TC ou encore ce qui peut faire partie du développement normal de l'enfant. À titre d'exemple, un répondant indique que : « en travaillant avec des enfants ayant des TED, presque tous les enfants pourraient concorder avec la définition des TC. II est difficile de départager ce qui est en lien avec les caractéristiques TED de ce qui l'est avec les TC ». Un autre répondant rapporte quant à lui qu'il est : " difficile en bas âge de départager ce qui fait partie du développement normal versus d'un TC, par exemple mordre à 2 ans versus à 5 ans ». Sur la difficulté à déterminer ce qui fait partie des TC, un troisième répondant pose la question suivante : "Comment différencier les TC des autres symptômes comme les problématiques sensorielles ou les malaises physiques? " Aussi, un participant conclut qu' : « il est encore difficile d'imaginer que quelqu'un avec un TED puisse avoir plus qu'un TED».

\section{Perceptions en regard de l'intégration des enfants ayant des TC en milieu de garde et en milieu scolaire}

La troisième question de recherche vise à décrire la perception des participants quant à l'intégration des enfants ayant des TC dans les milieux préscolaires. Plus spécifiquement, elle vise à documenter leurs observations quant à la présence d'enfants ayant un TSA et des TC dans les milieux de garde ou scolaire ordinaire et à identifier les défis qu'ils perçoivent face à leur intégration. Parmi l'ensemble des répondants, 40,1\% indiquent que les enfants ayant des TC avec lesquels ils travaillent ne sont pas inclus dans des milieux de garde réguliers tandis que $22,7 \%$ sont en milieu de garde régulier à temps plein. Dans une proportion non négligeable, $20,5 \%$ des répondants mentionnent que les enfants ont été intégrés puis retirés ; $13,6 \%$ signalent qu'ils sont intégrés en étant en tout temps supervisés par un adulte (un pour un) et $4,5 \%$ des répondants rapportent que ces enfants sont intégrés avec la présence d'un accompagnateur. Enfin, deux répondants affirment que les enfants ayant un TC sont systématiquement exclus des milieux de garde. Un répondant indique que : "L'intégration [en milieu de garde] d'un enfant TGC est quasi impossible parce qu'on doit penser à la sécurité des autres enfants ». En même temps, un autre répondant révèle que : «La mentalité est vraiment différente d'un milieu à l'autre. Dans les CPE, tous les enfants sont égaux et doivent recevoir les mêmes choses ». Concernant la collaboration entre les milieux de garde et les CRDITED pour le maintien de l'intégration, un répondant rapporte que : "Comme ils [les enfants] sont en liste d'attente sur une longue période [pour obtenir des services des CRDITED], ils ont déjà eu le temps de se faire exclure des CPE ». Un 
autre indique que : «Comme la plupart des enfants ne sont pas intégrés et restent à la maison, l'évaluation est plus difficile ».

On constate également qu'il y a des différences dans l'intégration des enfants ayant des TC en milieu de garde par rapport au milieu scolaire. Un répondant indique : "L'intégration se fait relativement bien en service de garde, mais il reste beaucoup de travail à faire en milieu scolaire (formation, collaboration, ouverture d'esprit, etc.) ». En ce sens, deux autres répondants mentionnent: "Les écoles spécialisées devraient être plus formées en TC/TGC parce que dès que l'enfant émet des comportements perturbateurs, il est retiré des écoles. " et "Les écoles spécialisées devront s'outiller elles aussi pour répondre aux besoins des enfants (encadrement, ratio, mesures de contrôle, etc.) ». Aussi, un participant soulève que : "Même lorsqu'on donne des outils à l'école, le personnel ne les utilise pas toujours et il arrive souvent que nos enfants soient dirigés vers des classes spécialisées. II faudrait qu'ils aient une meilleure formation sur notre clientèle (TED) ». II est mentionné que : "Le système de classement de la région n'encourage pas l'intégration en milieu régulier. Quand il y a présence de TC/TGC, les enfants sont orientés en classe ou école spécialisée ». Un autre répondant souligne que : « Nos stratégies d'intervention font que la personne est fonctionnelle en $\mathrm{ICl}$, mais que la marche est haute à 6 ans pour l'intégrer dans une classe. Les problèmes ressurgissent également dès que le programme $\mathrm{ICl}$ est terminé ». En ce sens, un intervenant mentionne que : « Les milieux scolaires n'adaptent pas leurs interventions à l'enfant et utilisent les mêmes interventions pour tous les enfants avec des TC, ce qui ne fonctionne pas. Ils ont besoin de plus de formation... ". Dans la même ligne de pensée, un répondant soulève que : "Les milieux d'intégration exigent les mêmes comportements de la part des enfants TC/TGC que de celle des enfants sans TC ». Concernant la collaboration entre les CRDITED et les écoles, un participant fait ressortir que lorsqu'un enfant ayant des TC est intégré : « L'école a la possibilité de nous appeler, mais ils ne le font pas. Ils ne semblent pas interpréter nos rapports de la même manière que nous ».

Tous les répondants (100\%) perçoivent des besoins sur le plan de l'intégration de ces enfants en milieux réguliers lorsqu'ils sont interrogés à ce sujet. Le Tableau 1 décrit les défis rapportés par les répondants par rapport à cette question. À noter que les besoins les plus souvent mentionnés sont la formation, le soutien direct aux milieux d'accueil et le besoin de personnel additionnel.

\section{La perception des rôles en CRDITED auprès des enfants ayant des TC}

Les répondants ont été interrogés sur leurs perceptions par rapport à leurs rôles auprès des enfants ayant des TC au sein de leur équipe. Le Tableau 2 fait état des rôles spontanément identifiés par les répondants dans les situations où les enfants présentent des TC. Le rôle le plus souvent cité est relié à l'intervention. La moitié des répondants, et parmi ceux-ci $73 \%$ sont des éducateurs, ont identifié tenir ce rôle dans les dossiers TC. Ce sont aussi les éducateurs qui rapportent le plus souvent assumer un rôle relié à l'ICI (54\%), contrairement aux professionnels qui le mentionnent peu (15\%). Seulement $7 \%$ des répondants affirment être impliqués dans la conception des plans d'intervention, soit $30 \%$ des professionnels. Le deuxième rôle le plus cité ( $22 \%$ des participants) est celui de soutien aux éducateurs, soit 
Tableau 1. Perceptions du personnel des CRDITED face aux défis à l'intégration des enfants ayant des TC

Manque de formation des milieux d'accueil

Manque de soutien pour le milieu d'accueil

Manque de personnel dans le milieu d'accueil

Non collaboration du milieu d'accueil

Manque de formation des intervenants

Volet comportemental priorisé sur le cognitif et autonomie lors des interventions

\begin{tabular}{cc}
$\mathrm{N}$ & $\%$ \\
\hline 30 & 68 \\
19 & 43 \\
7 & 16 \\
5 & 11 \\
4 & 9 \\
4 & 9
\end{tabular}

Manque de communication et de collaboration entre les milieux

Besoin d'accompagnement $1: 1$

Manque de soutien aux parents

Besoin d'un local de retrait

Orientation systématique en classe spécialisée lors de la présence de TC

Parents minimisent l'information de peur de perdre des services

Évaluation difficile parce que l'enfant reste à la maison

Nouveaux comportements problématiques lors de l'intégration

3

3

2

2

2

1

12

Sécurité des autres enfants

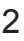

2

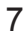

7

5

5

5

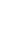

2

$\mathrm{N}=$ nombre de mentions

chez la moitié des professionnels et $60 \%$ des gestionnaires. Le rôle de soutien aux éducateurs est d'ailleurs le rôle le plus mentionné par les professionnels $(50 \%)$ avec celui de supervision (50\%). L'évaluation vient ensuite en seconde position d'importance pour les professionnels (40\%). Pour les gestionnaires, le rôle le plus nommé est celui relié à la gestion de l'équipe $(80 \%)$, suivi du rôle de soutien aux éducateurs $(60 \%)$. Vingt pour cent $(20 \%)$ de l'ensemble des participants reconnaissent avoir un rôle de soutien auprès de partenaires dont la famille, les milieux de garde et les milieux scolaires. À ce sujet, un répondant indique que le nombre d'heures dispensées à la famille diminue le soutien offert aux enfants. Les intervenants ont un nombre d'heures de services à donner au sein d'une famille et doivent partager ce temps entre les services directs à l'enfant et les services à sa famille. Seulement $20 \%$ des répondants ont identifié spontanément avoir un rôle relié à l'évaluation d'enfants ayant des TC. Par ailleurs, on ne rapporte aucune mention concernant l'évaluation fonctionnelle ou l'analyse multimodale.. 
Tableau 2. Rôles mentionnés spontanément par les répondants auprès des enfants ayant des troubles de comportement

\begin{tabular}{lcccccc}
\hline & \multicolumn{2}{c}{ Intervenant } & \multicolumn{2}{c}{ Professionnel } & \multicolumn{2}{c}{ Gestionnaire } \\
\cline { 2 - 6 } & $\mathrm{n}$ & $\%$ & $\mathrm{n}$ & $\%$ & $\mathrm{n}$ & $\%$ \\
Intervention & 19 & 73 & 2 & 20 & 1 & 20 \\
$\mathrm{ICI}$ & 14 & 54 & 1 & 10 & - & - \\
Soutien des éducateurs & 2 & 8 & 5 & 50 & 3 & 60 \\
Évaluation & 4 & 15 & 4 & 40 & 1 & 20 \\
Soutien à la famille & 8 & 31 & 1 & 10 & - & - \\
Support partenaires & 5 & 19 & 1 & 10 & - & - \\
(CPE, scolaire) & - & - & 5 & 50 & 1 & 20 \\
Supervision & 3 & 12 & 1 & 10 & 1 & 20 \\
Observation & - & - & - & - & 4 & 80 \\
Gestion d'équipe & - & - & 3 & 30 & - & - \\
Plan d'intervention & 2 & 8 & - & - & - & - \\
Accompagnement & - & - & 2 & 20 & - & - \\
Psychoéducation & - & - & - & - & 1 & 20 \\
Administration & & & & & & \\
\hline
\end{tabular}

\section{Discussion}

L'âge préscolaire est propice au développement de TC chez les enfants tout-venant et l'est encore davantage pour les enfants ayant des particularités développementales telles que les TSA (Baker, Blacher, Crnic, \& Edelbrock, 2002; Matson, Gonzalez, Wilkins, \& Rivet, 2008). Ces troubles sont une cible importante d'intervention précoce, voire de prévention, pour les établissements desservant ces enfants; et cela, d'autant plus que les TC peuvent s'exacerber avec le temps en l'absence d'intervention appropriée (Emerson \& Einfeld, 2010). De plus, la présence de ces troubles peut compliquer la réponse des enfants aux programmes d'intervention mis en place dans les établissements de services, notamment les services d'ICl en CRDITED pour les enfants ayant un TSA. La présente étude s'insère dans un programme de recherche qui s'intéresse à l'étude et à l'évaluation des interventions offertes aux enfants présentant à la fois une DI ou un TSA et un TC. Cette étude constitue une première étape de ce programme qui permet de documenter la perception des personnes travaillant en CRDITED quant aux TC chez les jeunes enfants dans le cadre de leur travail.

Malgré que des difficultés sur le plan de la reconnaissance des TC en petite enfance soient notées par les répondants, la majorité $(95,5 \%)$ de ceux-ci rapportent avoir travaillé au cours de la dernière année avec au moins un enfant de six ans et moins correspondant à la définition retenue. Aussi, près de $70 \%$ des répondants 
rapportent avoir travaillé avec plus d'un enfant ayant un TC. Ces données montrent l'importance de s'intéresser de façon spécifique aux comportements problématiques dans l'organisation des services d'ICI en CRDITED. Les intervenants doivent être formés aux différentes stratégies d'évaluation et d'intervention reliées aux comportements problématiques afin que celles-ci soient privilégiées lors des programmes d'ICl. Un deuxième article (Rivard, Morin, Dionne, \& Gagnon, soumis) rapportant les résultats concernant la formation, l'évaluation et l'intervention montre que la majorité des répondants des équipes $\mathrm{ICl}$ mentionnent avoir besoin de formation concernant l'évaluation fonctionnelle et les stratégies d'intervention pour des comportements problématiques. Ils se sentent effectivement peu outillés à intervenir auprès des enfants ayant des TC dans le cadre de leurs services d'ICI.

Concernant la deuxième question, soit les comportements problématiques observés par les répondants, les comportements les plus souvent mentionnés sont, par ordre d'importance : les comportements d'agression physique envers autrui; les comportements perturbateurs; les comportements d'automutilation; la non collaboration; l'agitation; les troubles de l'alimentation; les stéréotypies; les comportements de provocation; la difficulté dans la gestion des émotions; les troubles du sommeil; les problématiques en lien avec la propreté; les comportements destructeurs et les troubles anxieux. Ces résultats concordent avec ce qui est rapporté dans les études antérieures, soit celles qui présentent les TC les plus fréquents chez les enfants ayant un TSA ou celles faisant ressortir les priorités d'intervention identifiées par les familles (Horner et al., 2002; Machalicek et al., 2007; Matson \& Nebel-Schwalm, 2007). Ces informations sont importantes car elles constituent un point de repère pour guider les programmes de formation s'adressant aux intervenants travaillant auprès de cette population. Elles permettent aussi d'orienter les programmes d'intervention spécialisés en fonction des problématiques observées à même les services. Et surtout, ces résultats permettent d'orienter le développement d'outils d'évaluation des TC pour les jeunes enfants ayant un TSA en identifiant les catégories comportementales à inclure dans ces outils. Néanmoins, les résultats concernant les comportements problématiques identifiés par les répondants soulèvent des interrogations. En effet, certains comportements rapportés par les répondants pourraient davantage faire partie des causes des TC, de comportements déficitaires en TSA ou encore de caractéristiques contribuant à établir ce diagnostic. C'est le cas par exemple des stéréotypies et de la gestion des émotions. Cela confirme les difficultés mentionnées par les répondants lorsqu'ils doivent faire des distinctions entre les caractéristiques des TSA et les TC. Un outil d'évaluation spécifique aux TC et adapté aux enfants ayant un TSA pourrait permettre de raffiner l'évaluation de la présence ou de l'absence d'un TC.

Pour la majorité des éducateurs, le rôle qu'ils rapportent assumer le plus souvent auprès des enfants ayant un TC est relié à l'intervention. Par contre, peu d'éducateurs mentionnent spontanément être impliqués dans la conception des plans d'intervention de ces enfants. Un peu plus de la moitié de ceux-ci $(54 \%)$ mentionnent avoir un rôle en lien avec l'ICI lorsqu'il s'agit d'enfants ayant des TC inscrits dans leurs services. Peu de professionnels et de gestionnaires rapportent avoir un rôle lié à l'intervention directe. 
Très peu de répondants mentionnent spontanément être impliqués dans le processus d'évaluation ou d'observation, soit $20 \%$. Ce sont principalement les professionnels $(40 \%)$ qui perçoivent remplir ce rôle, comparativement aux gestionnaires $(20 \%)$ et intervenants $(15 \%)$. L'évaluation fonctionnelle des comportements problématiques et l'intervention adaptée à cette évaluation sont pourtant des composantes essentielles des programmes découlant de l'AAC tels que les programmes d'ICI (voir notamment Leaf \& McEachin, 1999; Maurice, 1996). Les données de la présente enquête montrent que peu de répondants mentionnent spontanément avoir ce rôle auprès des enfants ayant un TC. Quoique les données puissent être expliquées en partie par la structure du questionnaire (questions ouvertes), elles montrent l'importance de mettre davantage l'accent sur l'évaluation fonctionnelle et l'intervention spécifique entourant les TC dans les programmes d'ICI dans les CRDITED. Certains gestionnaires ont par ailleurs mentionné ne pas avoir reçu de formation clinique en TC ou en petite enfance puisque leur rôle est davantage lié à la gestion.

Peu de répondants ont mentionné avoir un rôle de soutien aux partenaires $(13,6 \%)$ ou à la famille (20\%), quoique ce type de soutien ait été mentionné comme un réel besoin, comme le suggèrent les études réalisées auprès des familles. Ces études font notamment ressortir des lacunes sur le plan du partenariat dans les services offerts à leur enfant. Cet écart entre les besoins de soutien et l'offre de services de soutien peut s'expliquer par différents facteurs. Dans le cas du soutien aux familles, il peut entre autres s'expliquer par le fait que les parents et les personnes de l'entourage minimisent la gravité ou l'impact des TC ou encore qu'ils n'en parlent pas du tout, par crainte de perdre des services. Plusieurs répondants ont mentionné que les parents ne révèlent ces informations que lorsqu'ils ont créé un lien avec les membres de l'équipe, puisqu'ils ont par le passé déjà perdu des services (p. ex., une place en milieu de garde) en mentionnant les problématiques de leur enfant. Ces résultats peuvent aussi s'expliquer par l'organisation des services d'ICI en CRDITED ou l'organisation des mandats des différentes lignes de services du réseau de la santé et des services sociaux. Actuellement, l'organisation des services de réadaptation en CRDITED destinés à la petite enfance sont presque exclusivement dédiés à l'intervention directe auprès de l'enfant. Ce type de service est primordial et doit impérativement être maintenu. Néanmoins, les données sur le stress parental, la qualité de vie des familles et les besoins de services des familles d'enfants ayant un TSA (voir Rivard, Parent-Boursier, Terroux, \& Mercier, soumis; Sabourin, des Rivières-Pigeon \& Granger, 2011) convergent vers l'importance d'offrir également des services directs à la famille. Ces services doivent être distincts des heures dédiées à l'enfant et poursuivent des objectifs différents. Les données sur les rôles perçus mettent en lumière l'importance de mieux définir les rôles et les responsabilités au sein des équipes dans le cas où les enfants présentent des TC. Chaque étape du processus clinique doit être prise en charge par une personne responsable de cette étape qui s'en approprie l'implantation et s'assure de son efficacité.

En ce qui concerne l'intégration des enfants ayant des TC, près de $40 \%$ des répondants rapportent que ces enfants ne sont pas intégrés en milieu de garde, alors que $23 \%$ des répondants sont d'avis qu'ils le sont. Les autres répondants mentionnent que les enfants sont exclus des milieux de garde mais après avoir 
connu une période d'intégration. D'autres précisent que les enfants ayant des TC sont physiquement dans les milieux de garde mais qu'ils sont constamment supervisé par un adulte. Cela dit, tous les répondants notent des besoins sur le plan de l'intégration, soit plus particulièrement en ce concerne la formation et le soutien aux milieux d'accueil ainsi que la collaboration entre les CRDITED et ces milieux d'accueil. L'intégration en milieu scolaire est également perçue plus complexe que celle en milieu de garde. Ces résultats relatifs à l'intégration vont dans le sens des études mentionnées précédemment réalisées auprès des familles, où les parents rapportent que leurs enfants sont souvent exclus des services (McGill, Papachristoforou, \& Cooper, 2006; Wodehouse \& McGill, 2009).

Cette étude comporte également trois limites. La première a trait à l'utilisation d'un échantillon de convenance. Aussi, il faut mentionner que cette étude fait état de la perception des participants par rapport au nombre d'enfants qu'ils ont reçus dans leurs services et qui présentent des TC. La consigne donnée aux participants était d'évaluer dans leurs dossiers combien d'enfants correspondaient à la définition de Tassé et ses collaborateurs (2010). II faut donc interpréter les résultats avec précaution puisqu'il ne s'agit pas ici d'une étude dans laquelle des outils d'évaluation des TC ont été utilisés pour estimer le nombre d'enfants ayant des TC et à qui on dispense des services. Mentionnons néanmoins que ceci n'était pas l'objectif de l'étude et qu'à notre connaissance, il n'existe pas d'outil standardisé adapté à cette clientèle et disponible en français. Notons par ailleurs que la définition utilisée pour identifier les enfants qui présentent des TC/TGC dans le cadre de cette étude a été développée plus particulièrement pour une clientèle adulte. Cette définition, qui a servi de point de repère aux répondants, pourrait être moins adaptée aux enfants, particulièrement dans le cas où l'on voudrait également cibler les comportements problématiques qui peuvent mener à des TC.

En conclusion, cette première étude sur la perception du personnel des CRDITED par rapport aux TC chez les jeunes enfants fait ressortir l'importance d'étudier directement le phénomène et de proposer aux milieux cliniques de la formation adéquate ainsi que des outils d'évaluation et d'intervention adaptés à cette population. Les services d'ICl ont été récemment implantés dans les CRDITED du Québec et nous avons peu d'information sur les différents aspects reliés à la formation des éducateurs et les professionnels, sur les paramètres de services implantés (intensité, durée, milieu d'intervention) et sur les stratégies privilégiées dans les plan d'intervention. Les données de la présente enquête montrent que les milieux perçoivent des besoins de formation en analyse appliquée du comportement et en évaluation fonctionnelle. Ces formations permettraient au personnel de mettre en place des interventions plus individualisées, selon le profil de chacun des enfants. La formation, les paramètres de services et les stratégies d'intervention sont au coeur de tout programme d'ICI, particulièrement lorsque ces programmes sont offerts aux enfants ayant un TSA et un TC. De façon générale, la littérature actuelle et les résultats de l'étude montrent le besoin d'aborder les comportements problématiques sous l'angle du dépistage et de la prévention, tant pour la recherche que pour la pratique. En effet, on n'insistera jamais assez sur le fait que sans intervention précoce et appropriée, les comportements problématiques se cristallisent dans le temps jusqu'à devenir des TC. 


\section{Références}

Allen, D., \& Tynan, H. (2000). Responding to aggressive behavior: Impact of training on staff members' knowledge and confidence. Mental Retardation, 38 (2), 97-104.

Baker, B. L., Blacher, J., Crnic, K., \& Edelbrock, C. (2002). Behavior problems and parenting stress in families of three-year old children with and without developmental delays. American Journal on Mental Retardation, 107, 433-444.

Berryman, J., Evans, I. M., \& Kalbag, A. (1994). The effects of training in nonaversive behavior management on the attitudes and understanding of direct care staff. Journal of Behavior Therapy and Experimental Psychiatry, 25 (3), 241-250.

Emerson, E., \& Einfeld, S. (2010). Emotional and behavioural difficulties in young children with and without developmental delay: A bi-national perspective. Journal of Child Psychology and Psychiatry, 51, 583-593.

Farmer, C. A., \& Aman, M. G. (2011). Aggressive behaviour in a sample of children with autism spectrum disorders. Research in Autism Spectrum Disorders, 5, 317-323.

Fava, L., \& Strauss, K. (2011). Crosssetting complementary staff- and parent-mediated early intensive behavioral intervention for young children with autism: A research-based comprehensive approach. Research in Autism Spectrum Disorders, 5, 512522.

Fédération québécoise des centres de réadaptation en déficience intellectuelle et troubles envahissants du développement (FQCRDITED) (2010). Rapport annuel 2009-2010. www. fqcrdi.qc.ca

Fox, L., Dunlap, G., \& Cushing, L. (2002). Early intervention, positive behaviour support, and transition to school. Journal of Emotional and Behavioral Disorders, 3, 149-157.
Gamache, V., Joly, J., \& Dionne, C. (2011). La fidélité et qualité d'implantation du programme québécois d'intervention comportementale intensive destiné aux enfants ayant un trouble envahissant du développement en CRDITED. Revue de psychoéducation, 40, 1-23.

Gurney, J. G., McPheeters, M. L., \& Davis, M. M. (2006). Parental report of health conditions and health care use among children with and without autism: National Survey of Children's Health. Archives of Pediatrics and Adolescent Medicine, 160 (8), 825.

Hastings, R. P. (1997). Measuring staff perceptions of challenging behaviour: The Challenging Behaviour Attributions Scale (CHABA). Journal of Intellectual Disability Research. Special Issue: Mental Health and Intellectual Disability, 41 (6), 495-501. doi: 10.1111/j.13652788.1997.tb00742.x

Hastings, R. P., \& Brown, T. (2002). Behavior problems of children with autism, parental self-efficacy, and mental health. American Journal on Mental Retardation, 107 (3), 222-232. doi: 10.1352/0895-8017(2002)107

Hastings, R. P. (2005). Staff in special education settings and behaviour problems: Towards a framework for research and practice. Educational Psychology, 25 (2-3), 207-221. doi: $10.1080 / 0144341042000301166$

Horner, R.H., Carr, E.G., Strain, P.S., Todd, A.W., \& Reed, H.K. (2002). Problem behavior interventions for young children with autism: $A$ research synthesis. Journal of Autism and Developmental Disorders, 32, 423-446.

Icasiano, F., Hewson, P., Machet, P., Cooper, C., \& Marshall, A. (2004). Childhood autism spectrum disorder in the Barwon region: a community based study. Journal of paediatrics and child health, 40 (12), 696-701. 
Jacobs, M \& Woolfson, L. (2012). Parents' Causal. Beliefs and Causal Attributions for Misbehaviour in Children with ID: The Relationship between Control and Parenting Strategies. In a Paper Sessions on Behavioral Problems at 9th biennial international conference of European Conference on Intellectual and developmental Disabilities, Trieste, Italy.

Jang, J., Dixon, D. R., Tarbox, J., \& Granpeesheh, D. (2011). Symptoms severity and challenging behavior in children with ASD. Research in Autism Spectrum Disorders, 5, 1028-1032.

Leaf, R., \& McEachin, J. (1999). A Work in Progress. DRL Books, New York.

Machalicek, W., O'Reilly, M.F., Beretvas, N., Sigafoos, J., \& Lancioni, G.E. (2007). A review of interventions to reduce challenging behaviour in school settings for children with autism spectrum disorders. Research in Autism Spectrum Disorders, 1, 229-246.

Mahan, S., \& Maston, J. (2011). Convergent and discriminant validity of the Autism Spectrum Disorder-Problem Behavior for Children (ASD-PBC) against the Behavioral Assessment System for Children, Second Edition (BASC2). Research in Autism Spectrum Disorders, 5, 222-229.

Makrygianni, M. K., \& Reed, P. (2010). A meta-analytic review of the effectiveness of behavioural early intervention programs for children with Autistic Spectrum Disorders. Research in Autism Spectrum Disorders, 4 (4), 577-593.

Matson, J.L., Gonzalez, M., \& Rivet, T.T. (2008). Reliability of the autism spectrum disorder-behavior problems for children (ASD-BPC). Research in Autism Spectrum Disorders, 2, 696706.

Maston, J. L., \& Nebel-Schwalm, M. (2007). Assessing challenging behaviors in children with autism spectrum disorders: A review. Research in Developmental Disabilities, 28, 567-579.
Maston, J., Wilkins, J., \& Macken, J. (2009). The relationship of challenging behaviors to severity and symptoms of autism spectrum disorders. Journal of Mental Health Research in Intellectual Disabilities, 2, 29-44.

Maurice, C., Green, G, \& Luce, S. C. (1996). Behavioral. Intervention for Young Children with Autism. PRO-ED inc.

McGill, P., Papachristoforou, E., \& Cooper, V. (2006). Support for family carers of children and young people with developmental disabilities and challenging behaviour. Child: Care, Health and Development, 32 (2), 159165.

Mitchell, G., \& Hastings, R. P. (2001). Coping, burnout, and emotion in staff working in community services for people with challenging behaviors. American Journal on Mental Retardation, 106 (5), 448-459. doi: 10.1352/08958017(2001)106

Morgan, G. M., \& Hastings, R. P. (1998). Special educators' understanding of challenging behaviours in children with learning disabilities: s Sensitivity to information about behavioural function. Behavioural and Cognitive Psychotherapy, 26 (1), 43-52.

Rivard, M. (2011). Progression des comportements verbaux et sociaux et les effets de la sensibilité sociale chez des enfants ayant un trouble dans le spectre de l'autisme inscrits à un programme d'intervention comportementale précoce. (Thèse de doctorat), Université du Québec à Montréal.

Rivard, M., \& Forget, J. (2012). Verbal behavior in young children with autism spectrum disorders at the onset of an early behavioral intervention program. The Psychological Record, 62, 165-186.

Rivard, M., Forget, F., Giroux, G., Regli, G., \& Kerr, K., (soumis). Children with Autism Spectrum Disorders: Social and Verbal Behavior Changes During Intervention. Manuscrit soumis pour publication. 
Rivard, M., Parent-Boursier, C., Terroux, A., \& Mercier, C. (submitted). Determinants of stress in parents of children with autism spectrum disorders. Manuscript submitted for publication.

Sabourin, G., des Rivières-Pigeon, C., \& Granger, S. (2011). L'intervention comportementale intensive, une affaire de famille? Analyse du regard que posent les mères d'enfants autistes sur leurs intervenantes. Revue de psychoéducation, 40, 51-70.

Symes, M.D., Remington, B., Brown, T., \& Hasting, R. P. (2006). Early intensive behavioral intervention for children with autism therapists' perspectives on achieving procedural fidelity. Research in Developmental Disabilities, 27, 30-42.
Tassé, M.J., Sabourin, G., Garcin, N., \& Lecavalier, L. (2010). Définition d'un trouble grave du comportement chez les personnes ayant une déficience intellectuelle. Canadian Journal of Behavioural Science, 42, 62-69.

Wodehouse, G., \& McGill, P. (2009). Support for family carers of children and young people with developmental disabilities and challenging behaviour: What stops it being helpful? Journal of Intellectual Disability Research, 53, 644-653. 